\title{
Ecological and population characteristics of the seashell Conus princeps on the Pacific coast of central Mexico
}

\section{Atributos ecológicos y poblacionales del caracol Conus princeps en la parte central de la costa mexicana del Pacífico}

\author{
Jesús Emilio Michel-Morfín ${ }^{1 *}$, Gilberto A Medina-Vargas ${ }^{1}$, Víctor Landa-Jaime ${ }^{1}$, \\ Judith Arciniega-Flores ${ }^{1}$, Manuel B Aguilar ${ }^{2}$, Edgar P Heimer de la Cotera ${ }^{2}$ \\ 1 Departamento de Estudios para el Desarrollo Sustentable de Zonas Costeras, Centro Universitario de Costa \\ Sur-Universidad de Guadalajara, Valentín Gómez Farías 82, CP 48980, San Patricio-Melaque, Jalisco, \\ Mexico. \\ 2 Instituto de Neurobiología, Universidad Nacional Autónoma de México, Blvd. Juriquilla 3001, CP 76230, \\ Juriquilla, Quintana Roo, Mexico. \\ * Corresponding author. Email: emilio.michel@academicos.udg.mx
}

\begin{abstract}
Marine snails of the genus Conus have acquired remarkable biomedical importance because of the high number of toxins they produce for feeding and self-defense. One of these toxins gave way to a new medication and two other are under development for clinical and cosmetic purposes. Nevertheless, there is little basic knowledge about this group of species, particularly in Mexico. This study aimed to determine the relative abundance, spatial distribution, bathymetric distribution, and habitat preference of the snail Conus princeps along the southern coast of Jalisco (Mexico). To achieve this goal, we conducted direct samplings by snorkeling or scuba diving at 13 beaches, covering the intertidal, shallow subtidal, and deep subtidal zones. Relative density was between 0.021 and 0.418 snails per $100 \mathrm{~m}^{2}$, with an average value of 0.152 snails per $100 \mathrm{~m}^{2}$. The population showed an aggregated spatial distribution pattern according to the nearest neighbor index. Snails had shells that measured between $23.1 \mathrm{~mm}$ and $52.2 \mathrm{~mm}$ long, with an average shell size of $39.7 \mathrm{~mm}$. Conus princeps was mainly distributed in the intertidal and shallow subtidal zones. The smaller organisms were present at shallower depths, while the larger organisms were distributed throughout the sampled depth habitats. In general, snail abundance decreased as depth increased. From the underwater photography analysis, we determined that Conus princeps snails prefer rocky bottom habitats covered with brown seaweed.
\end{abstract}

Keys words: mollusk, cone snails, ecology, Conus princeps, Pacific Ocean.

RESUMEN. Los caracoles marinos del género Conus han adquirido notable importancia biomédica por el elevado número de toxinas que producen para su alimentación y defensa, de las cuales una ha dado lugar a un nuevo fármaco y otras más se encuentran en desarrollo para aplicaciones clínicas y cosméticas. No obstante, es poco el conocimiento básico que se tiene sobre este grupo de especies, en particular en México. Con este trabajo se busca determinar la abundancia relativa, distribución espacial, distribución batimétrica y preferencia por el sustrato del caracol Conus princeps a lo largo de la costa de Jalisco (México). Para ello, se realizaron búsquedas directas por medio de buceo libre o autónomo en 13 playas cubriendo los estratos del intermareal, submareal somero y submareal profundo. La densidad relativa presentó valores de entre 0.021 y 0.418 caracoles por $100 \mathrm{~m}^{2}$ y un promedio de 0.152 caracoles por $100 \mathrm{~m}^{2}$. La población presentó una distribución espacial de tipo agregada según los análisis del índice del vecino más cercano. Se presentaron caracoles con conchas de entre los $23.1 \mathrm{~mm}$ y $52.2 \mathrm{~mm}$ de largo, con un promedio de $39.7 \mathrm{~mm}$. Conus princeps se distribuyó principalmente en los estratos del intermareal y submareal somero. Los organismos de tallas menores se presentaron a bajas profundidades, mientras que los organismos de tallas mayores se distribuyeron en todo el estrato de profundidad muestreado. En general, se observó que con el aumento de la profundidad, disminuyó la abundancia de los caracoles. A partir del análisis de fotografías submarinas, se determinó que existe una preferencia de los caracoles C. princeps por habitar en sustrato rocoso cubierto de algas pardas.

Palabras clave: moluscos, caracoles cono, ecología, Conus princeps, océano Pacífico.

\section{INTRODUCTION}

Cone snails, so called after the shape of their shells, constitute a genus of the Conidae family (Prosobranchia: Neogastropoda). They are considered the most diverse mollusk genus (Ponder 1973, Kohn 1990, Bandyopadhyay et al. 2008), with 803 extant species (WoRMS 2018) and 841 fossil species (Duda and Kohn 2005). Cone snails are found mainly in tropical oceans, from the subtidal zone to $1,000-\mathrm{m}$ depth, and inhabit various types of substrate, such as rock,

\section{INTRODUCCIÓN}

Los caracoles cono, llamados así por la forma de su concha, constituyen un género de la familia Conidae (Prosobranchia: Neogastropoda). Son considerados el género de moluscos más diverso (Ponder 1973, Kohn 1990, Bandyopadhyay et al. 2008), con 803 especies actualmente reconocidas (WoRMS 2018) y 841 especies fósiles (Duda y Kohn 2005). Los conos se distribuyen principalmente en los océanos tropicales, desde la zona submareal hasta los 1,000 $\mathrm{m}$ de profundidad, 
sand, mud, and seabed covered with vegetation or coral reefs (Dance and von Cosel 1977, Díaz-M et al. 2005). The species studied here, Conus princeps Linnaeus, 1758, is distributed from the Gulf of California, Mexico, to the northern coast of Peru (Keen 1971).

The evolutionary success of cone snails is attributed mainly to their sophisticated feeding method, which allows them to efficiently hunt using a modified radula to inject paralyzing toxins (conotoxins) into their prey (Nybakken 1970, López 2001). To hunt, cone snails use their venom apparatus, which produces venom and includes the injection mechanism (Marshall et al. 2002). Conotoxins are peptides that, after being synthesized in the venom apparatus, are injected through the chitinous, hypodermic needlelike radular teeth generated in the radular sac (Marshall et al. 2002). These toxins have been studied since the 1960s, when their pharmacological potential was discovered (Khon 1956). Up until 200780 medical patents were derived from these toxins (Kohn and Anderson 2010). At present, there is one drug, called Prialt, that is synthesized from the venom of the snail Conus magus Linnaeus, 1758 and is used on patients who experience intense pain (EMA 2014).

Particularly for C. princeps, Medina-Elizalde (2012) found that the species produces toxins with capacity to block ion channels. Gorrostieta-Hurtado et al. (2012) analyzed the effect of venom extracts from 3 different parts of the venom duct and found different regions of toxin biosynthesis. Zamora-Bustillos et al. (2014) and Morales-González et al. (2015) characterized conotoxins from 3 Conus species on the coast of Jalisco (Mexico), including C. princeps. More recently, Bernáldez et al. (2016) isolated a new conotoxin from this species.

Despite all the above, there are few studies on the ecology and biology of C. princeps on the Pacific coast of Mexico. In Oaxaca, Medina-Elizalde (2010) described the morphology of C. princeps and Conus brunneus. On the other hand, in Guerrero, Flores-Garza et al. (2014) conducted a study on the family Conidae, including its distribution, abundance, size composition, and association with rocky substrates, but only recorded one C. princeps specimen. Ortíz-Arellano (2014) determined species richness and the biological, ecological, and anatomical aspects for 31 Conoidea species on the coast of Sinaloa, and reported that Conidae was the most representative family with 17 species, including C. princeps; only 3 C. princeps specimens were collected.

For the Pacific coast of central Mexico, Landa-Jaime et al. (2013) recorded the presence of C. princeps and 7 other species of the genus Conus associated with the Tenacatita coral reef, Jalisco. For the soft bottoms on the continental shelf of Jalisco and Colima, Michel-Morfín et al. (2014) recorded 9 species of the genus Conus, including C. princeps. Bastida-Izaguirre (2014) made out an inventory of invertebrates and fish in Chamela Bay, Jalisco, and highlighted the presence of 4 mollusk species of the genus Conus: C. brunneus, Conus nux, C. princeps, and Conus purpurascens. In the y habitan diversos tipos de sustrato, como fondos rocosos, arenosos, fangosos y cubiertos de vegetación y arrecifes de coral (Dance y von Cosel 1977, Díaz-M et al. 2005). La especie en estudio, Conus princeps Linnaeus, 1758, se distribuye desde el golfo de California en México hasta el norte de Perú (Keen 1971).

El éxito evolutivo de los caracoles cono recae principalmente en el sofisticado método de alimentación, que les permite cazar muy eficientemente utilizando una rádula modificada a través de la cual inyectan toxinas (conotoxinas) capaces de paralizar a sus presas (Nybakken 1970, López 2001). Para cazar, los caracoles cono utilizan su aparato venenoso, donde se genera el veneno y se encuentra el mecanismo de inyección (Marshall et al. 2002). Las conotoxinas son péptidos que, una vez sintetizados en el aparato de veneno, se inyectan a través de dientes quitinosos que tienen forma de aguja hipodérmica y que se generan en el saco radular (Marshall et al. 2002). Estas toxinas han sido objeto de estudios desde la década de 1960, cuando se descubrió su potencial farmacológico (Kohn 1956). Hasta 2007, se habían desprendido de éstas 80 patentes médicas (Kohn y Anderson 2010). Actualmente, existe un fármaco, llamado Prialt, sintetizado a partir del veneno del caracol Conus magus Linnaeus, 1758 , y es utilizado en pacientes con dolores muy intensos (EMA 2014).

En particular para C. princeps, Medina-Elizalde (2012) encontró que la especie produce toxinas con capacidad para bloquear canales iónicos. Gorrostieta-Hurtado et al. (2012) analizaron el efecto del extracto venenoso contenido en 3 partes diferentes del conducto venenoso, y observaron que existen diferentes regiones de biosíntesis de toxinas. Zamora-Bustillos et al. (2014) y Morales-González et al. (2015) caracterizaron las conotoxinas de 3 especies de Conus de la costa de Jalisco (México), entre ellas las de C. princeps. Recientemente, Bernáldez et al. (2016) aislaron una nueva conotoxina de esta especie.

No obstante lo anterior, son pocos los estudios que se han realizado sobre la ecología y biología de C. princeps en la costa mexicana del Pacífico. En Oaxaca, Medina-Elizalde (2010) realizó la descripción morfológica de C. princeps y Conus brunneus. Por su parte, Flores-Garza et al. (2014), en Guerrero, realizaron un estudio sobre la familia Conidae y su asociación con el sustrato rocoso, su distribución, su abundancia y su composición de tallas, pero solo registraron un ejemplar de C. princeps. Para la costa de Sinaloa, Ortíz-Arellano (2014) determinó la riqueza específica y los aspectos biológicos, ecológicos y anatómicos de 31 especies de conoideos, y destacó que la familia Conidae fue la más representativa con 17 especies, entre ellas C. princeps, de la cual solo recolectaron 3 ejemplares.

En particular para la parte central de la costa mexicana del Pacífico, Landa-Jaime et al. (2013) registraron la presencia de C. princeps y otras 7 especies del género Conus asociadas al arrecife coralino de Tenacatita, Jalisco. Michel-Morfín et al. (2014), para fondos blandos de la plataforma continental de 
Pacific transition zone off Mexico, García-Hernández (2014) recorded 8 Conidae species in Point Tehuamixtle, close to Cabo Corrientes, Jalisco, and found very low densities of C. princeps. In a recent study, Cortez-Orozco (2017) found 5 Conus species in the subtidal zone on the coast of Jalisco.

Although there are some studies on this group of sea snails, the present study aims to determine particular ecological and population attributes for $C$. princeps along the southern coast of Jalisco to help expand knowledge on this species and provide elements to determine if preservation and protection measures are needed for populations of $C$. princeps, given its biomedical potential.

\section{MATERIALS AND METHODS}

The southern coast of Jalisco is part of marine ecoregion no. 17, which corresponds to the Mexican Pacific Transition (Wilkinson et al. 2009), and marine priority region no. 26 (Chamela-El Palmito) (Arriaga-Cabrera et al. 1998). The region has a narrow continental shelf measuring approximately $2,364 \mathrm{~km}^{2}$. Ocean current circulation in this zone is characterized by 3 events: one from February to April, with high influence of cold waters from the California Current; another from August to January, with warm waters from the Equatorial Countercurrent; and the third from May to July, with a mixing period when the 2 currents converge (Wyrtki 1965). These events produce dynamic water circulation and, consequently, variability in oceanographic conditions in the zone (Silva-Segundo et al. 2008, Ambriz-Arreola et al. 2012).

Thirteen sampling sites were selected from Navidad Bay $\left(19^{\circ} 10^{\prime} 30^{\prime \prime} \mathrm{N}, \quad 104^{\circ} 41^{\prime} 30^{\prime \prime} \mathrm{W} ; \quad 1^{\circ} 12^{\prime} 50^{\prime \prime} \mathrm{N}, \quad 104^{\circ} 42^{\prime} 45^{\prime \prime} \mathrm{W}\right)$ to Chamela Bay $\left(19^{\circ} 21^{\prime} 11^{\prime \prime} \mathrm{N}, 105^{\circ} 01^{\prime} 43^{\prime \prime} \mathrm{W} ; 1^{\circ} 38^{\prime} 06^{\prime \prime} \mathrm{N}\right.$, $\left.105^{\circ} 12^{\prime} 30^{\prime \prime} \mathrm{W}\right)$. Four direct searches of Conus snails were performed at each of the 13 sites (Fig. 1) at the lowest tides of the day between February 2001 and January 2014. Searches were performed in the rocky intertidal zone (0-1 m depth), shallow subtidal zone (1-5 m depth), and deep subtidal zone (5-20 m depth). Different subaquatic techniques were used depending on depth, such as snorkeling and scuba diving. All sampling areas were delimited using a GPS MAP60CSx (Garmin; Olathe, Kansas).

To determine snail distribution and abundance in the intertidal zone, a direct scanning survey was carried out, and total area coverage was delimited with the GPS data. Every time a snail was found, a $0.25-\mathrm{m}^{2} \mathrm{PVC}$ quadrant equipped with a $10-\mathrm{cm}$ graduated scale and a ScubaPro depthometer was placed around the snail. A submarine photograph was taken with a Canon SD 1100 digital camera equipped with a Canon polycarbonate case. The condition of the organism (alive or dead) and data about its location (type of environment where it was found, depth, and number of individuals) were recorded. When complete organisms were found (shell and soft body), snails were collected in numbered plastic bags to obtain biometric data and determine the species. This process was repeated for samplings in the shallow and
Jalisco y Colima, registraron 9 especies del género Conus, entre las cuales se encontró C. princeps. Bastida-Izaguirre (2014) realizó un inventario de invertebrados y peces de la bahía de Chamela, Jalisco, y destacó entre los moluscos la presencia de 4 especies del género Conus: C. brunneus, Conus nux, C. princeps y Conus purpurascens. Para la zona de transición del Pacífico frente a México, García-Hernández (2014) registró 8 especies de cónidos en punta Tehuamixtle, parte cercana a cabo Corrientes, Jalisco, y encontró densidades muy bajas de C. princeps. Recientemente, CortezOrozco (2017) encontró 5 especies de Conus en el submareal de la costa de Jalisco.

Si bien existen algunos estudios sobre este grupo de caracoles, en este trabajo se pretende conocer los atributos ecológicos y poblacionales particulares de C. princeps a lo largo de la costa sur de Jalisco, lo cual permitirá acrecentar el conocimiento de esta especie y contar con elementos para determinar si es necesario establecer medidas de protección o conservación de las poblaciones, aspecto importante dado su potencial uso biomédico.

\section{MATERIALES Y MÉTODOS}

La costa sur de Jalisco forma parte de la ecorregión marina no. 17, que corresponde al Pacífico Transicional Mexicano (Wilkinson et al. 2009) y a la región marina prioritaria no. 26 (Chamela-El Palmito) (Arriaga-Cabrera et al. 1998). La plataforma continental de esta región es estrecha, con un área aproximada de $2,364 \mathrm{~km}^{2}$. La circulación de las corrientes en esta zona se caracteriza por 3 eventos: uno de febrero a abril, con un mayor efecto de aguas frías por la corriente de California; otro de agosto a enero, con aguas cálidas de la contracorriente ecuatorial; y el tercero de mayo a julio, con un periodo de mezcla en el que convergen las 2 corrientes (Wyrtki 1965). Estos eventos producen una circulación dinámica y, en consecuencia, variabilidad en las condiciones oceanográficas de la zona (Silva-Segundo et al. 2008, Ambriz-Arreola et al. 2012).

Se seleccionaron 13 sitios de muestreo desde bahía de Navidad $\left(19^{\circ} 10^{\prime} 30^{\prime \prime} \mathrm{N}, \quad 104^{\circ} 41^{\prime} 30^{\prime \prime} \mathrm{W} ; 1^{\circ} 12^{\prime} 50^{\prime \prime} \mathrm{N}\right.$, $\left.104^{\circ} 42^{\prime} 45^{\prime \prime} \mathrm{W}\right)$ hasta bahía de Chamela $\left(19^{\circ} 21^{\prime} 11^{\prime \prime} \mathrm{N}\right.$, $\left.105^{\circ} 01^{\prime} 43^{\prime \prime} \mathrm{W} ; 1^{\circ} 38^{\prime} 06^{\prime \prime} \mathrm{N}, 105^{\circ} 12^{\prime} 30^{\prime \prime} \mathrm{W}\right)$. Entre febrero de 2011 y enero de 2014 se realizaron 4 búsquedas directas de caracoles Conus en cada uno de los 13 sitios (Fig. 1) durante los periodos de mareas más bajas del día. Estas búsquedas se llevaron a cabo en las zonas del intermareal rocoso $(0 \mathrm{a}$ $1 \mathrm{~m}$ de profundidad), submareal somero ( $1 \mathrm{a} 5 \mathrm{~m}$ de profundidad) y submareal profundo (5 a $20 \mathrm{~m}$ de profundidad). En función de la profundidad, se utilizaron técnicas subacuáticas, como buceo libre y autónomo. Todas las áreas consideradas fueron delimitadas por medio de un posicionador satelital GPS MAP60CSx (Garmin; Olathe, Kansas).

Para establecer la distribución y abundancia de los caracoles en la zona intermareal, se realizó una búsqueda directa en forma de barrido, y se delimitó el área total recorrida con los datos del GPS. Cada vez que se encontró un organismo, 


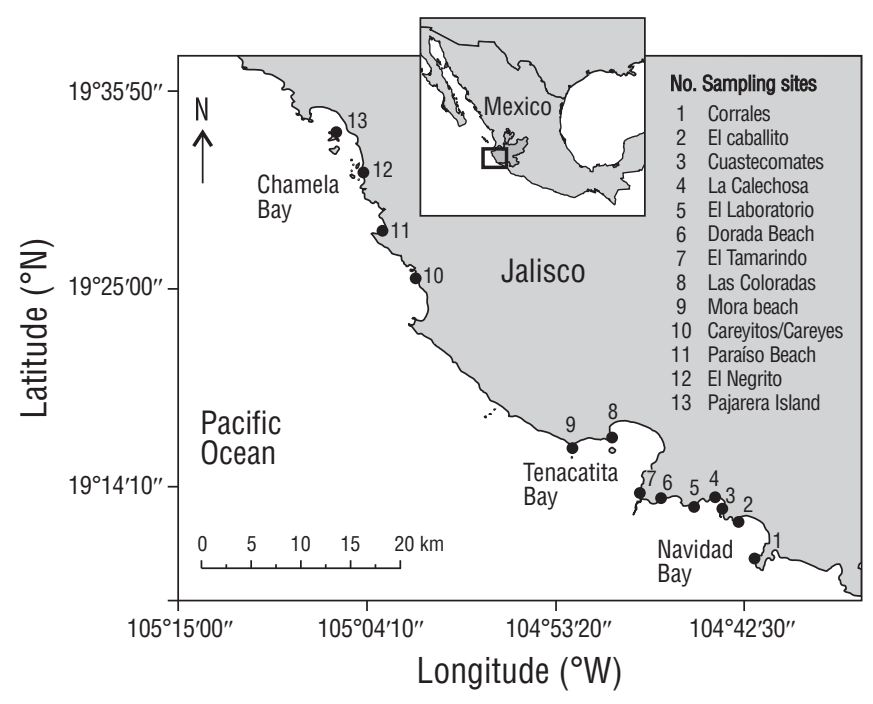

Figure 1. Sites on the southern coast of Jalisco (Mexico) where Conus princeps was sampled.

Figura 1. Sitios en la costa sur de Jalisco (México) donde se realizaron los muestreos de Conus princeps.

deep subtidal zones, except that a diver pulling a GPS buoy at the surface followed the bubbles of the 2 divers searching for snails to record the collection point. When visibility and depth conditions hindered visual communication, one of the divers inflated and released a colored balloon to signal that an organism had been found.

The process of recording biometric data consisted in measuring the maximum length and width of the shell $(\mathrm{mm})$ with a digital vernier. Weight $(\mathrm{g})$ was recorded with a scale that had a capacity of $100 \mathrm{~g}$. This process was done aboard the vessel or on the beach in the supralittoral zone. To determine the general structure of the $C$. princeps population, biometric data was used to obtain average snail size at each site, a general size-frequency histogram, and the length-weight relationship.

For the spatial dispersion and relative density analyses, all GPS position records for C. princeps organisms were considered. Satellite images and ArcView 3.3 and ArcGis 9.3 (ESRI 2011) software were used to obtain the geographic location of each snail, total area sampled, and, with this, relative density (expressed as the number of organisms per unit area). The sampling polygon was obtained on a map that was created by assembling the satellite image and a hexagonal grid of $100-\mathrm{m}^{2}$ cells.

A Friedman analysis of variance by ranks (nonparametric test) was done to determine significant differences in abundance data by depth stratum and between sampling sites. In addition, a Fisher's nonparametric $F$-test was done (Zar 2010). These 2 tests minimized the risk of type I and II errors.

On the other hand, the nearest neighbor method was used to determine the spatial pattern for $C$. princeps at each se colocó un cuadrante de PVC de $0.25 \mathrm{~m}^{2}$, que incorporaba una escala graduada de $10 \mathrm{~cm}$ y un profundímetro ScubaPro, alrededor del caracol. Se tomó una fotografía submarina con una cámara digital Canon SD 1100 provista de una carcasa de policarbonato de la misma marca. Se registró el estado del organismo (vivo o muerto) y los datos de su localización (tipo de ambiente donde se encontró, profundidad y número de individuos). En caso de tratarse de un organismo completo (concha y parte blanda), los caracoles fueron recolectados en una bolsa plástica numerada para, posteriormente, registrar los datos biométricos y determinar la especie. Para el submareal somero y profundo, se repitió el proceso anterior, excepto que se implementó un sistema en el que un buzo en la superficie llevaba un GPS sobre una boya y seguía el rastro de las burbujas de la pareja de buzos que realizaban la búsqueda de los caracoles para registrar el punto de recolecta. Cuando las condiciones de visibilidad y profundidad no permitían la comunicación visual, uno de los buzos inflaba y liberaba un globo de colores, como señal de que había encontrado un organismo.

El proceso de registro de datos biométricos consistió en medir el largo y ancho máximo de la concha $(\mathrm{mm})$ por medio de un vernier digital. Se registró el peso $(\mathrm{g})$ con una báscula con capacidad de $100 \mathrm{~g}$. Este proceso se llevó a cabo a bordo de la embarcación de apoyo o en la zona supralitoral de la playa. Los datos biométricos se utilizaron para determinar la estructura general de la población de C. princeps, ya que se obtuvo la talla promedio para cada sitio, un histograma general de frecuencia de tallas y la relación longitud-peso.

Para el análisis de dispersión espacial y densidad relativa, se tomó en cuenta la totalidad de los registros de las posiciones GPS de organismos de C. princeps. Por medio de imágenes satelitales y el software ArcView 3.3 y ArcGis 9.3 (ESRI 2011), se obtuvo el punto geográfico correspondiente a cada caracol, el área total muestreada y, a partir de ésta, la densidad relativa (expresada como el número de organismos por unidad de área). El polígono de muestreo se obtuvo mediante la elaboración de un mapa, ensamblando la imagen satelital y un enmallado de hexágonos con área de $100 \mathrm{~m}^{2}$.

Para determinar si existían diferencias significativas en los datos de abundancia por estrato de profundidad y entre sitios de muestreo, se realizó un análisis de varianza por rangos de Friedman (prueba no paramétrica). Adicionalmente, se realizó la prueba no paramétrica $F$ de Fisher (Zar 2010). A partir de estas 2 pruebas, se minimizó el riesgo de errores tipo I y II.

Por otra parte, para determinar el tipo de dispersión espacial de C. princeps en cada uno de los sitios de muestreo, se utilizó el método del vecino más cercano. Este método tiene la bondad de permitir determinar, a partir del cálculo de las distancias entre los organismos, un índice que indica si la distribución espacial es agregada (valor cercano a 0), al azar (valor cercano a 1) o uniforme (valor mayor que 1). El valor se obtiene directamente de la subrutina de análisis presente en el programa ArcGis 9.3 (ESRI 2011). 
sampling site. This method allows using the measurements of distance between organisms to determine an index, that indicates if spatial distribution is clustered (value near 0 ), random (value near 1), or uniform (value greater than 1). The value is obtained directly from the subroutine analysis in the ArcGis 9.3 program (ESRI 2011).

Environments with C. princeps individuals were characterized using the underwater digital images obtained during fieldwork. Percent substrate coverage was determined by analyzing the image with the Coral Point Count v.4.2 computer program (Kohler and Gill 2006).

\section{Results}

A total of 180 specimens of C. princeps were recorded at the 13 sampling sites along the southern coast of Jalisco. Conus princeps was more abundant at 3 sampling sites: Mora Beach (39 sea snails), Cuastecomates (34 sea snails), and El Laboratorio (24 sea snails). The highest abundance per stratum was observed in the shallow subtidal zone at Mora Beach (22 sea snails) and the deep subtidal zone at Cuastecomates (19 sea snails) (Table 1). Average sea snail abundance considering all beaches was $13.84 \pm 6.31$, with a maximum of 39 sea snails and a minimum of 4 sea snails.
A partir de las fotografías digitales submarinas obtenidas en el trabajo de campo, se caracterizó el ambiente en el que se presentó el caracol C. princeps. Se determinó el porcentaje de cobertura de cada tipo de sustrato analizando la imagen con el programa de cómputo Coral Point Count v.4.2 (Kohler y Gill 2006).

\section{RESUltados}

Se logró registrar un total de 180 organismos de C. princeps en los 13 sitios de muestreo considerados en la costa sur de Jalisco. Se registraron 3 sitios con mayor abundancia, playa Mora (39 caracoles), Cuastecomates (34 caracoles) y El Laboratorio (24 caracoles). La mayor abundancia por estrato se encontró en el submareal somero de playa Mora (22 caracoles) y en el submareal profundo de Cuastecomates (19 caracoles) (Tabla 1). La abundancia promedio de caracoles estimada para todas las playas fue de $13.84 \pm 6.31$, con una máxima de 39 caracoles y una mínima de 4 caracoles.

Para calcular la densidad relativa de caracoles por estrato de playa y para el total de cada playa, se generaron mapas con los datos de campo georeferenciados para cada uno de los sitios. Por cuestiones de espacio, se muestra solo el mapa correspondiente a la playa El Laboratorio, ubicado dentro

Table 1. Conus princeps abundance and relative population density by sampling site and zone.

Tabla 1. Abundancia y densidad relativa de Conus princeps por sitio y zona de muestreo.

\begin{tabular}{lcccccccc}
\hline & \multicolumn{2}{c}{ Intertidal } & \multicolumn{2}{c}{ Shallow subtidal } & \multicolumn{2}{c}{ Deep subtidal } & & Total \\
Site & Abundance & Density* & Abundance & Density* & Abundance & Density* & abundance & density* \\
\hline Corrales & 0 & 0 & 3 & 0.141 & 2 & 0.320 & 5 & 0.153 \\
El Caballito & 0 & 0 & 6 & 0.282 & 8 & 0.330 & 14 & 0.293 \\
Cuastecomates & 3 & 0.097 & 12 & 0.165 & 19 & 0.593 & 34 & 0.25 \\
La Calechosa & 4 & 0.036 & 1 & 0.015 & 0 & 0 & 5 & 0.021 \\
El Laboratorio & 3 & 0.924 & 11 & 0.337 & 10 & 0.466 & 24 & 0.418 \\
Dorada Beach & 1 & 0.102 & 7 & 0.094 & 4 & 0.163 & 12 & 0.11 \\
El Tamarindo & 1 & 0.018 & 4 & 0.026 & 1 & 0.015 & 6 & 0.022 \\
Las Coloradas & 0 & 0 & 1 & 0.038 & 3 & 0.0312 & 4 & 0.105 \\
Mora Beach & 12 & 0.039 & 22 & 0.094 & 5 & 0.085 & 39 & 0.064 \\
Careyitos/Careyes & 1 & 0.020 & 7 & 0.063 & 1 & 0.012 & 9 & 0.037 \\
Paraiso Beach & 0 & 0 & 7 & 0.040 & 0 & 0 & 7 & 0.027 \\
El Negrito & 1 & 0.369 & 8 & 0.225 & 1 & 0.233 & 10 & 0.235 \\
Pajarera Island & 0 & 0 & 3 & 0.259 & 8 & 0.243 & 11 & 0.246 \\
\hline
\end{tabular}

* Individuals per $100 \mathrm{~m}^{2}$. 
For relative density of sea snails per beach stratum and the total per beach, maps were generated with the georeferenced field data for each sampling site. Because of space limitation, the only map shown is that for the site that showed the highest relative density $(0.418$ sea snails per $100 \mathrm{~m}^{2}$ ), El Laboratorio Beach (Fig. 2), which is located inside Cuastecomates Cove. Results showed high variability in relative density estimates for the different beaches, with values between 0.021 and 0.418 sea snails per $100 \mathrm{~m}^{2}$ and an average of 0.152 sea snails per $100 \mathrm{~m}^{2}$ (Table 1).

The Friedman analysis of variance by ranks did not show significant differences between the densities at each stratum and the sampling site $\left(\chi^{2} r=2\right.$ and $\left.\chi^{2} r_{0.05,3,13}=6\right)$. Fisher's $F$-test showed coinciding results, given that the $F_{f}$ value was lower than $F_{0.05(1) 2,12}\left(F_{f}=1\right.$ and $\left.F_{0.05(1) 2,12}=3.8\right)$.

Sea snails in the $C$. princeps population on the southern coast of Jalisco measured between 23.1 and $52.5 \mathrm{~mm}$. The population was composed of mainly sea snails with shells measuring between 35.5 and $41.5 \mathrm{~mm}$ total length. The mode for total length was $41.0 \mathrm{~mm}$ (33 sea snails) (Fig. 3). Average length for all sampled sea snails was $39.7 \mathrm{~mm} \pm 0.84$ $(n=172)$.

Variability was observed in the average size of sea snails per sampling site. La Calechosa showed the highest average size per sampling site, with an average sea snail size of $46.4 \mathrm{~mm}$. Dorada Beach showed the minimum average size with $36 \mathrm{~mm}$ (Table 2). No trend was observed relating size with the latitude of sampling sites.

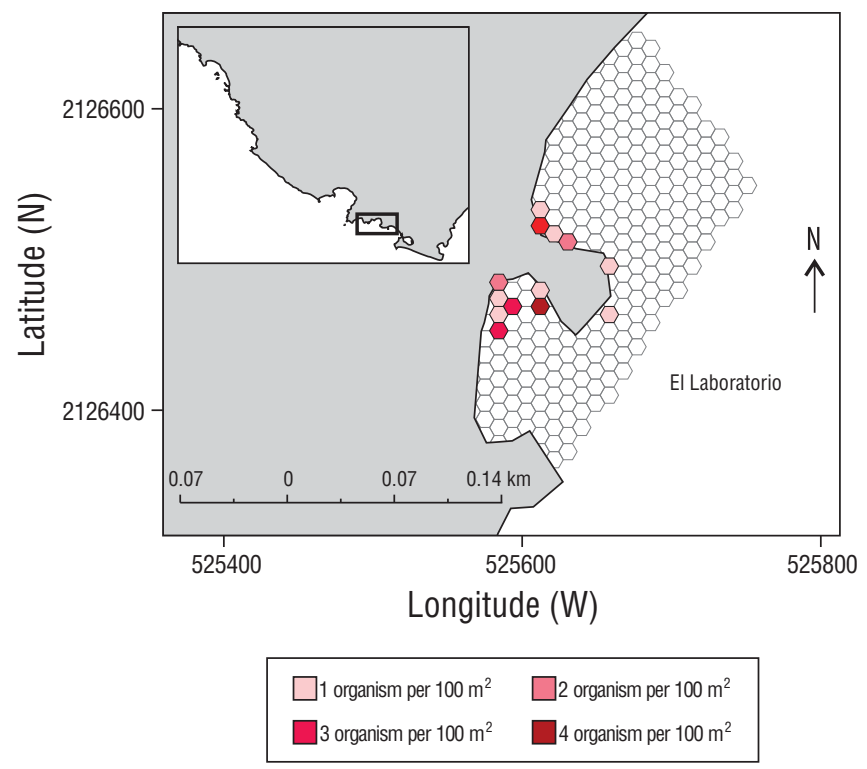

Figure 2. Map showing the relative density of Conus princeps at El Laboratorio.

Figura 2. Mapa que muestra la densidad relativa del caracol Conus princeps en El Laboratorio. de la caleta Cuastecomates, sitio que presentó la mayor densidad relativa (0.418 caracoles por $100 \mathrm{~m}^{2}$ ) (Fig. 2). Los resultados mostraron una amplia variabilidad en la densidad relativa calculada para las diferentes playas, con valores de entre 0.021 y 0.418 caracoles por $100 \mathrm{~m}^{2}$ y un promedio de 0.152 caracoles por $100 \mathrm{~m}^{2}$ (Tabla 1 ).

El análisis de varianza por rangos de Friedman no mostró diferencias significativas entre las densidades de cada estrato y el sitio de muestreo $\left(\chi^{2} r=2\right.$ y $\left.\chi^{2} r_{0.05,3,13}=6\right)$. La prueba $F$ de Fisher presentó resultados coincidentes, dado que el valor de $F_{f}$ fue menor que $F_{0.05(1) 2,12}\left(F_{f}=1\right.$ y $\left.F_{0.05(1) 2,12}=3.8\right)$.

En la población de C. princeps en la costa sur de Jalisco se encontraron caracoles que midieron entre 23.1 y $52.5 \mathrm{~mm}$ de longitud total. La población estuvo conformada principalmente por caracoles que se distribuyeron entre los $35.5 \mathrm{y}$ $41.5 \mathrm{~mm}$ de longitud total de la concha. La moda se presentó a los $41.0 \mathrm{~mm}$ de longitud total (33 caracoles) (Fig. 3). La talla promedio para todos los caracoles recolectados fue de $39.7 \mathrm{~mm} \pm 0.84(n=172)$.

Se observó variabilidad en las tallas promedio por sitio de muestreo. En La Calechosa se encontraron caracoles con una talla promedio de $46.4 \mathrm{~mm}$, la más alta de las tallas promedio encontradas por estación. La talla promedio mínima se registró en playa Dorada con $36 \mathrm{~mm}$ (Tabla 2). No se observó tendencia alguna con relación a la talla y la latitud del sitio de muestreo.

En cuanto a la dispersión espacial de C. princeps, el índice del vecino más cercano indicó que la mayoría de los sitios $(53.8 \%)$ presentaron una distribución tipo agregada; no obstante, se observaron otros sitios en los cuales la dispersión de los caracoles fue uniforme $(30.8 \%)$ o aleatoria $(15.4 \%)$ (Tabla 2).

El diagrama de dispersión con los datos de la relación talla-peso mostró un valor de $b$ muy cercano a tres (2.9) y un buen ajuste del modelo de regresión potencial $\left(r^{2}=0.80\right.$, $n=172$ ), por lo que se puede considerar que los caracoles presentan un crecimiento de tipo alométrico (Fig. 4).

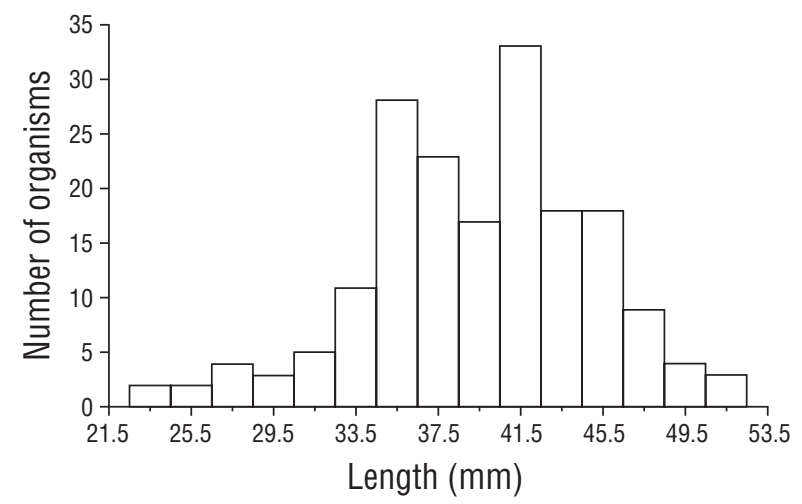

Figure 3. Histogram showing size-fequency distribution for Conus princeps on the southern coast of Jalisco.

Figura 3. Histograma de distribución de frecuencia de talla de Conus princeps en la costa sur de Jalisco. 
Table 2. Nearest neighbor index value and length measurements for Conus princeps by sampling site.

Tabla 2. Valor del índice del vecino más cercano y medidas de longitud para Conus princeps por sitio de muestreo.

\begin{tabular}{lccccc}
\hline Site & $\begin{array}{c}\text { Nearest } \\
\text { neighbour index }\end{array}$ & $\begin{array}{c}\text { Type of } \\
\text { distribution }\end{array}$ & $\begin{array}{c}\text { Maximum } \\
\text { length }(\mathrm{mm})\end{array}$ & $\begin{array}{c}\text { Minimum } \\
\text { length }(\mathrm{mm})\end{array}$ & $\begin{array}{c}\text { Average } \\
\text { length (mm) }\end{array}$ \\
\hline Corrales & 3.68 & Uniform & 45.9 & 28.7 & 37.2 \\
El Caballito & 0.69 & Clustered & 52.5 & 30.3 & 39.9 \\
Cuastecomates & 0.66 & Clustered & 49.5 & 35.9 & 44.0 \\
La Calechosa & 11.61 & Uniform & 49.0 & 41.5 & 46.4 \\
El Laboratorio & 0.82 & Random & 52.5 & 23.1 & 38.6 \\
Dorada Beach & 0.44 & Clustered & 48.0 & 25.6 & 36.0 \\
El Tamarindo & 2.11 & Uniform & 46.5 & 23.8 & 39.6 \\
Las Coloradas & 3.52 & Uniform & 45.0 & 36.7 & 39.8 \\
Mora Beach & 0.49 & Clustered & 49.0 & 26.7 & 37.1 \\
Careyitos/Careyes & 0.41 & Clustered & 46.0 & 38.0 & 42.0 \\
Paraíso Beach & 0.60 & Clustered & 45.0 & 35.4 & 41.2 \\
El Negrito & 0.78 & Random & 46.0 & 31.5 & 38.9 \\
Pajarera Island & 0.65 & Clustered & 45.0 & 30.0 & 38.1 \\
\hline
\end{tabular}

The nearest neighbor index showed that for most sampling sites (53.8\%) the spatial distribution of $C$. princeps was clustered; nonetheless, sea snail distributions for some sites was uniform $(30.8 \%)$ or random (15.4\%) (Table 2$)$.

The dispersion diagram with the size-weight relationship data showed a $b$ value very close to three (2.9) and a good fit to the potential regression model $\left(r^{2}=0.80, n=172\right)$; therefore, we can say sea snails showed an allometric growth pattern (Fig. 4).

The assessment of the total number of sea snails in the depth interval considered in this study $(0-20 \mathrm{~m})$ showed that individuals of the $C$. princeps population on the southern coast of Jalisco were distributed between 0 and $15.6 \mathrm{~m}$ depth and were more abundant from 0 to $4 \mathrm{~m}$ depth at all sampling sites. Different models were tested, and the best fit was obtained with the negative exponential model, which showed a determination coefficient of 0.82 . This relation showed that the presence of sea snails decreased with depth (Fig. 5). Another observable trend was that larger organisms $(>32.5 \mathrm{~mm})$ were distributed at all depths between 0 and $16 \mathrm{~m}$ (Fig. 6, red rectangle), whereas smaller organisms were limited to $<9 \mathrm{~m}$ depths (Fig. 6, blue rectangle).

A total of 90 underwater images were processed and analyzed with the Coral Point Count software. Images showed that $C$. princeps individulas were found on 7 substrate types. Once the images were processed, the rock-with-algae substrate was determined as the preferential habitat (68\%) (Fig. 7), followed by seashell debris and sand (10\%), rocks with barnacles $(8 \%)$, sand (6\%), coral (4\%), mixed substrates (3\%), and gravel (1\%) (Fig. 7).
$\mathrm{Al}$ analizar el total de caracoles recolectados en el intervalo de profundidad considerado ( 0 a $20 \mathrm{~m}$ ), se observó que los individuos de la población de C. princeps en la costa sur de Jalisco se distribuyeron entre los 0 y $15.6 \mathrm{~m}$ de profundidad y tuvieron mayor presencia de los 0 a $4 \mathrm{~m}$ en todos los sitios. Se probaron diferentes modelos y el mejor ajuste se obtuvo con el modelo exponencial negativo, el cual presentó un coeficiente de determinación de 0.82 . De esta relación se pudo observar que cuando aumentó la profundidad, disminuyó la presencia de caracoles (Fig. 5). Otra tendencia que fue posible observar es que los organismos de tallas grandes $(>32.5 \mathrm{~mm})$ se distribuyeron en todas las profundidades entre los 0 y 16 m (Fig. 6, recuadro color rojo), mientras que la distribución de los organismos de tallas pequeñas se limitó a profundidades menores que $9 \mathrm{~m}$ (Fig. 6, recuadro color azul).

En total se procesaron y analizaron 90 fotografías submarinas con el software Coral Point Count. Se reconocieron 7 tipos de sustratos en los que se encontraban los organismos de C. princeps. Una vez procesadas las fotografías, se determinó que el hábitat preferente fue el sustrato de roca con alga (68\%) (Fig. 7), seguido de restos de conchas y arena (10\%), roca con balanos $(8 \%)$, arena $(6 \%)$, coral $(4 \%)$, mezcla de sustratos (3\%) y grava (1\%) (Fig. 7).

\section{DisCuSIón}

La población de C. princeps a lo largo de la costa sur de Jalisco ha sido poco estudiada, pues la mayoría de los trabajos previos consideran solo la presencia o ausencia de la especie en listados taxonómicos, o bien, son relativos a sus 


\section{Discussion}

There are few studies on the population of $C$. princeps on the southern coast of Jalisco, and most of the studies have focused solely on the presence or absence of the species in taxonomic lists or on their conotoxins. For the study area, Landa-Jaime (2013) and Cortez-Orozco (2017) mention the presence of 5 Conus species: C. brunneus, Conus gladiator, C. nux, C. princeps, and C. purpurascens. The same 5 species were observed in the present study, where $C$. princeps was the third most abundant with $17 \%$ of total abundance, only after C. nux (45\%) and C. brunneus (20\%). In the Tenacatita coral reef, Landa-Jaime (2013) found the same abundance order for the species, but showed important variations in percentages, with $C$. nux as the most abundant species representing $62 \%$ of total organisms and C. princeps showing only $4.7 \%$. Muñiz-Castillo (2014) mentioned that in the Carrizales coral reef, Colima (Mexico), C. nux was the most abundant species with $94.1 \%$ of total organisms and that $C$. princeps was the second most abundant with only $3.8 \%$. We consider that C. princeps abundance is medium to low, with respect to the other 5 species discussed in these studies.

In our study we were able to estimate relative density, but we had to use the number of sea snails per $100 \mathrm{~m}^{2}$ as a unit, indicating that the observed densities are very low. Densities for the C. princeps population on the southern coast of Jalisco varied but were low in general, since they ranged from 0.021 to 0.418 sea snails per $100 \mathrm{~m}^{2}$ (average of 0.152 sea snails per $100 \mathrm{~m}^{2}$ ). Landa-Jaime (2013) reported a density of 1.3 organisms per $200 \mathrm{~m}^{2}$ for the Tenacatita coral reef. Muñiz-Castillo (2014) observed that densities for the Carrizales coral reef

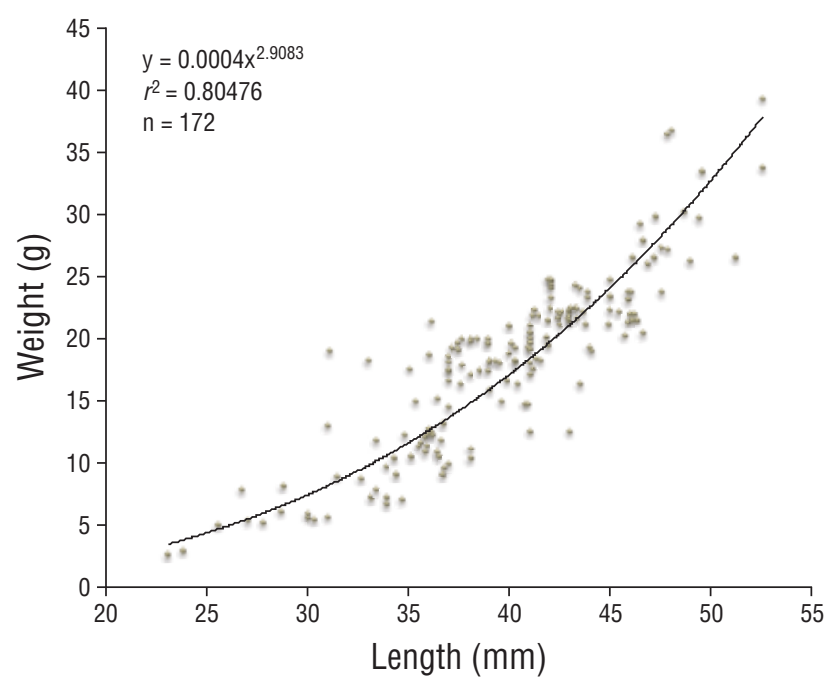

Figure 4. Length-weight relationship for Conus princeps on the southern coast of Jalisco.

Figura 4. Relación longitud-peso para el caracol Conus princeps en la costa sur de Jalisco. conotoxinas. Para la zona de estudio, Landa-Jaime (2013) y Cortez-Orozco (2017) mencionaron la presencia de 5 especies del género Conus: C. brunneus, Conus gladiator, C. nux, C. princeps y C. purpurascens. En el presente estudio se observaron las mismas 5 especies, de las cuales C. princeps fue la tercera especie más abundante con un $17 \%$ de la abundancia total, solo después de C. nux (45\%) y C. brunneus (20\%). En el arrecife de Tenacatita, Landa-Jaime (2013) encontró el mismo orden en la abundancia de las especies, pero con variaciones importantes en cuanto a los porcentajes, ya que C. nux fue la especie más abundante con el $62 \%$ del total de organismos encontrados y C. princeps con solo el 4.7\%. Muñiz-Castillo (2014) mencionó que, en el arrecife de Carrizales, Colima (México), C. nux fue la especie más abundante con el $94.1 \%$ del total de organismos encontrados y C. princeps la segunda más abundante con apenas el $3.8 \%$. Consideramos que C. princeps presenta abundancias de medias a bajas, con relación a las 5 especies referidas en estos trabajos.

Si bien en este trabajo se logró calcular la densidad relativa, fue necesario utilizar como unidad el número de caracoles por $100 \mathrm{~m}^{2}$, lo cual da una idea de que las densidades observadas son muy bajas. Las densidades que presenta la población de C. princeps en la zona sur de Jalisco son variables, pero en general son bajas, ya que variaron desde 0.021 hasta 0.418 organismos por $100 \mathrm{~m}^{2}$ (promedio de 0.152 caracoles por $100 \mathrm{~m}^{2}$ ). Landa-Jaime (2013) presentó una densidad de 1.3 organismos por cada $200 \mathrm{~m}^{2}$ para el arrecife coralino Tenacatita. Muñiz-Castillo (2014) observó diferentes densidades para el arrecife coralino Carrizales dependiendo del método de muestreo: para los recorridos lineales determinó una densidad de 0.55 organismos por $100 \mathrm{~m}^{2}$ y para los polígonos aleatorios determinó una densidad de 0.27 organismos por $100 \mathrm{~m}^{2}$. No obstante, la metodología utilizada en ambos estudios conlleva diferencias importantes, por lo que no es posible tener un punto de comparación real. Es importante unificar la metodología de muestreo utilizada para otros sitios del litoral del Pacífico para conocer el estado de la población de esta especie.

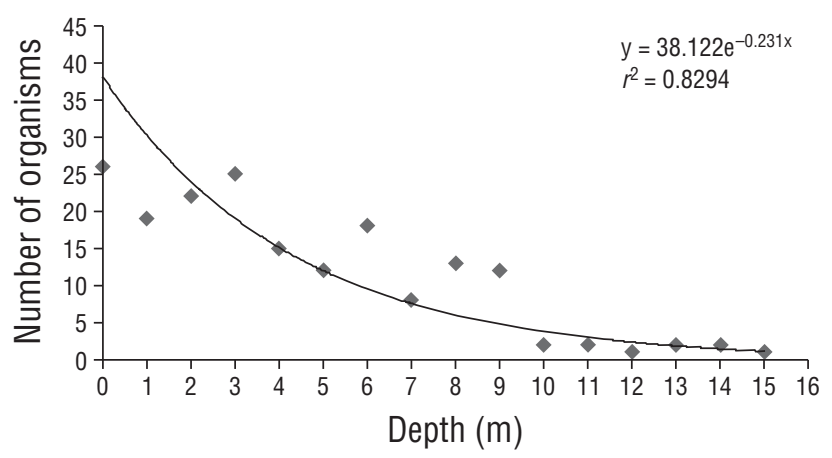

Figure 5. Conus princeps abundance with respect to depth.

Figura 5. Abundancia del caracol Conus princeps en función de la profundidad. 
were different depending on the used sampling method: a density of 0.55 organisms per $100 \mathrm{~m}^{2}$ was determined using linear transects and a density of 0.27 organisms per $100 \mathrm{~m}^{2}$ was determined using random polygons. However, the methodologies used in both studies result in important differences, and it is therefore not possible to have a true reference point. To determine the population status for this species, it is important to unify the sampling methods used for other sites on the Pacific coasts.

Size structure varied from 23.5 to $51.5 \mathrm{~mm}$ shell length, and the largest percentage of organisms was found in the 41.5-mm size class. The histogram observed in the present study is consistent que the size-frequency distribution histograms reported by Landa-Jaime (2013), who showed that the C. princeps population was delimited by total length sizes of 25 to $50 \mathrm{~mm}$ and that organisms measuring $40 \mathrm{~mm}$ in length were the most abundant. Gorrostieta-Hurtado et al. (2012) collected C. princeps specimens with average lengths of 45 and $50 \mathrm{~mm}$ in the Tenacatita coral reef and Cuastecomates, respectively, which is consistent with the observed sizes for organisms collected in the same region in the present study. Muñiz-Castillo (2014) recorded 26 to 46-mm sizes for $C$. princeps. Flores-Garza et al. (2014) reported a single organism measuring $31.5 \mathrm{~mm}$. In general, the sizes reported in the present study coincide with those reported in all the previous studies.

Size and weight data indicate that these sea snails exhibit allometric growth patterns, at least those in the observed size interval. It would be convenient to try to fit a growth model by using data from mark/recapture samplings or by tracking sizes across time to establish the relationship between the size and age of organisms and to determine juveniles sizes and recruitment periods, as has been done for other sea snail species in the intertidal zone (Michel-Morfín et al. 2000, Cervantes-Hernández et al. 2016).

Spatial dispersion is an important attribute for species because it can indicate mating aggregations, feeding aggregations, or aggregations that are dependent on prey microhabitats. On the other hand, in territorial species, spatial dispersion can indicate uniform distribution as a result of exclusion or competition. This was observed by Landa-Jaime (2013) for C. purpurascens, a piscivorous species that exhibits uniform distribution. In the case of $C$. princeps, the 3 types of spatial distribution were observed, but the most frequent one was the clustered type. This type of distribution could be explained by its feeding habits because, although it has not been observed feeding in the laboratory or the field, the shape of its radula suggests that it feeds on polychaetes (Ortíz-Arellano 2014). Therefore, sea snails could be aggregating around microhabitats that show optimum conditions for prey growth. Since sampling was done over a long period of time, changes in the observed distribution patterns between sampling sites could be explained by variations in the behavior of sea snails.

With respect to substrate affinity, C. princeps showed preference for rocks covered with algae $(68 \%)$. Ríos-Jara et

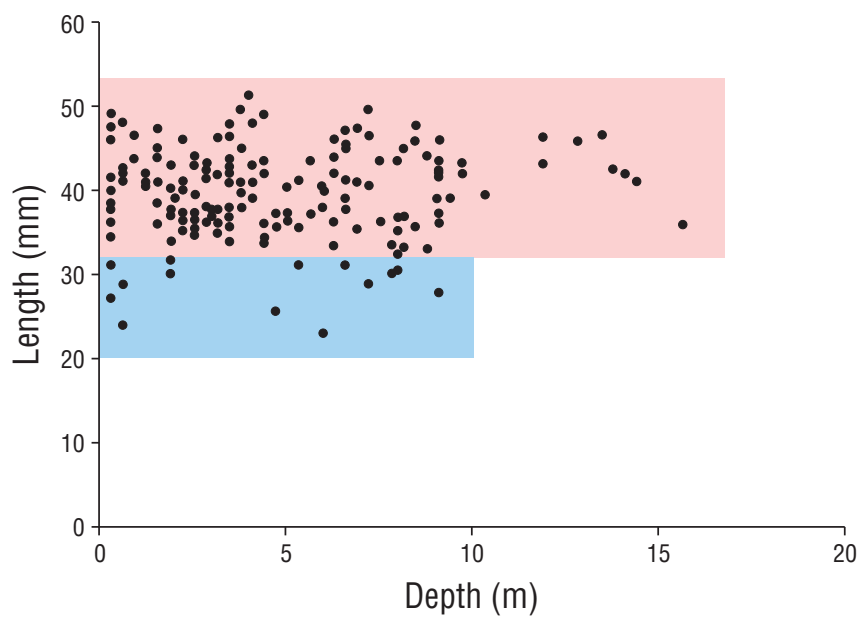

Figure 6. Observed relationship between sampling depth and Conus princeps size on the southern coast of Jalisco. Red, large individuals; blue, small inividuals.

Figura 6. Relación observada entre la profundidad de recolecta y la talla de los caracoles Conus princeps en la costa sur de Jalisco. Rojo, individuos grandes; azul, individuos pequeños.

La estructura de tallas varió de 23.5 a $51.5 \mathrm{~mm}$ de longitud de la concha, y el mayor porcentaje de los organismos se encontró en la clase de los $41.5 \mathrm{~mm}$. Los histogramas de distribución de frecuencias de tallas presentados por Landa-Jaime (2013) coinciden con el histograma observado en el presente trabajo, ya que mencionó que la población de C. princeps estuvo delimitada por tallas de 25 a $50 \mathrm{~mm}$ de longitud total y presentó mayor abundancia de organismos con tallas de $40 \mathrm{~mm}$. Gorrostieta-Hurtado et al. (2012) recolectaron organismos de C. princeps con longitud promedio de 45 y $50 \mathrm{~mm}$ en el arrecife coralino de Tenacatita y Cuastecomates, respectivamente, lo que concuerda con los organismos recolectados en la misma región en el presente trabajo. Muñiz-Castillo (2014) registró tallas de 26 a $46 \mathrm{~mm}$ para $C$. princeps. FloresGarza et al. (2014) observó una talla de $31.5 \mathrm{~mm}$ para el único organismo encontrado. Por tanto, en general, las tallas que se reportan en el presente estudio concuerdan con las reportadas en todos los trabajos realizados.

Los datos de tallas y pesos indican que estos caracoles crecen de manera alométrica, al menos en el intervalo de tallas observado. Resultaría muy adecuado intentar ajustar un modelo de crecimiento aplicando métodos de marcado y recaptura o a través del seguimiento de las tallas en el tiempo para poder establecer la relación entre la talla y la edad de los organismos y establecer la talla y época de reclutamiento de los juveniles, tal como ha sido establecido para otras especies de caracoles marinos de la zona intermareal (Michel-Morfín et al. 2000, Cervantes-Hernández et al. 2016).

La dispersión espacial es un atributo importante para las especies ya que puede indicar agregaciones reproductivas, agregaciones alimenticias o agregaciones en función de los microhábitats de sus presas. Por el contrario, en el caso de especies territorialistas, la dispersión espacial puede indicar 

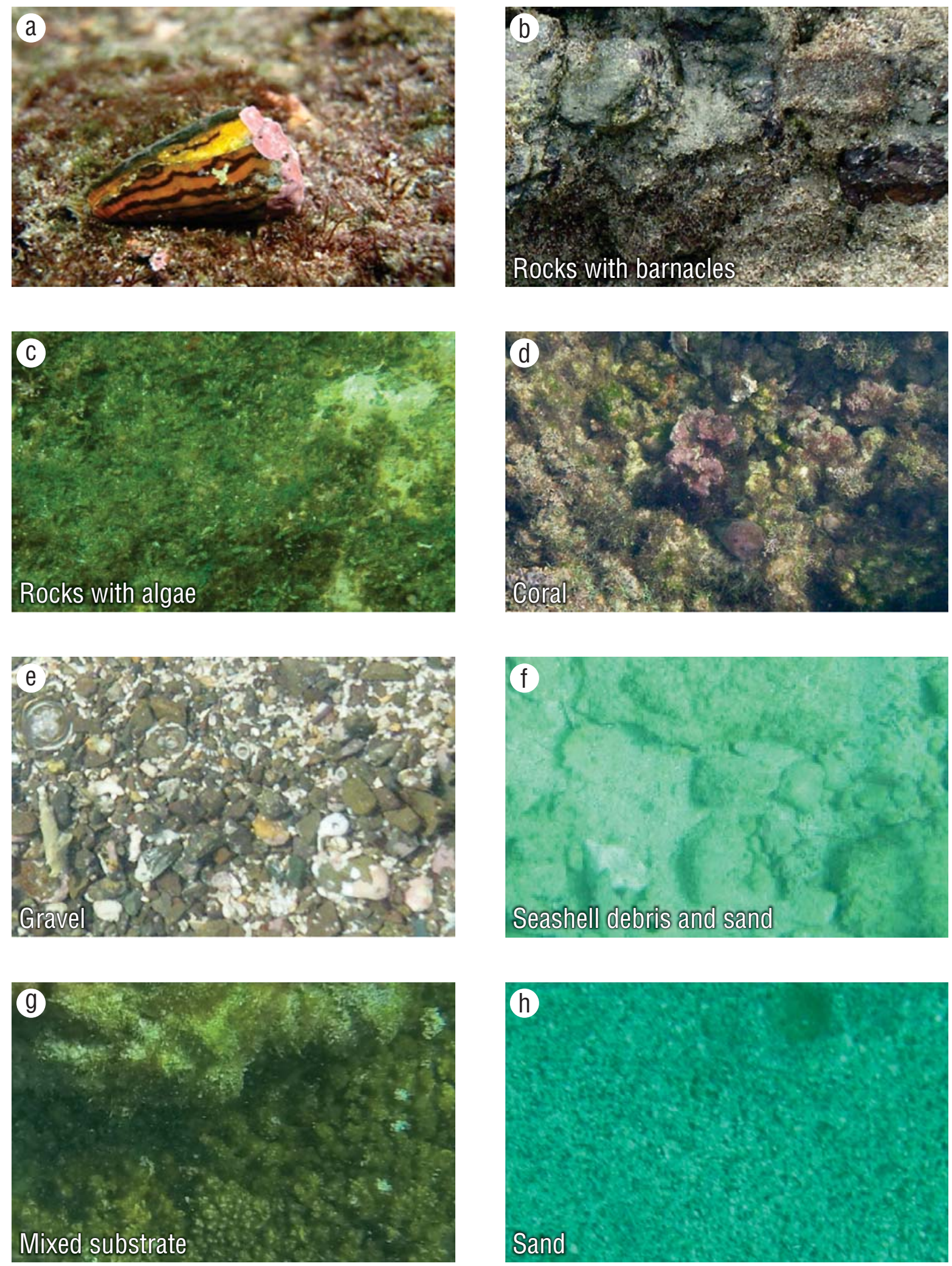

Figure 7. Marine snail Conus princeps Linnaeus, 1758 in the wild (a) and the substrate types considered in the study (b-h). Figura 7. Caracol Conus princeps Linnaeus, 1758 en su medio natural (a) y tipos de sustratos considerados (b-h). 
al. (2006) mentioned that this species is associated with rocky substrates and rocks covered with algae. Landa-Jaime (2013) mentioned that $C$. princeps preferred 3 substrate types and that it was most abundant (57\%) on coral substrates; however, the study was carried out at the Tenacatita coral reef. We have to consider that substrate preference could be determined by prey availability, the ease of going unnoticed while hunting, or the low chances of being hunted. To avoid bias in sampling site selection, we considered various sites along a latitudinal gradient with different characteristics; therefore, we believe that the results obtained in the present study are representative of the population on the southern coast of Jalisco.

The information presented in this study will contribute to knowledge on the sea snail C. princeps. Nevertheless, more studies on the biology of this species are needed because relevant aspects such as its reproductive season, growth, recruitment, and precise feeding habits, among others, have been scarcely studied and are necessary, together with specific studies on its toxicology, to learn more about this natural resource.

It is worth mentioning that after carrying out field activities at 13 sampling sites along the southern coast of Jalisco, which included 3 different environments and a bathymetric range between 0 and $20 \mathrm{~m}$ depth, only 180 live specimens of $C$. princeps were collected, indicating that this species is not abundant or is hard to find. This could be a reason for considering the establishment of long-term conservation measures for this species, such as restricting extraction of individuals or determining reserve zones or national parks. For sea snails of the genus Conus, in general, knowledge on the properties and biomedical applications of their toxins has shown advancements; however, other basic aspects, such as their distribution, abundance, basic population biology, and true number of species in Mexican waters, should also be studied.

\section{ACKNOWLedgments}

This work study received financial support from the Mixed Funds Project CONACYT-Government of the State of Jalisco (no. 2009-05-124426). GM received a BSc thesis grant from the same fund. We thank Israel Muñiz, Daniel Kosonoy, and Álvaro Reyes Espinoza for their support during field activities. We thank the anonymous reviewers for their valuable observations.

\section{English translation by Claudia Michel-Villalobos.}

\section{REFERENCES}

Ambriz-Arreola I, Gómez-Gutiérrez J, Franco-Gordo MC, Lavaniegos BE, Godínez-Domínguez E. 2012. Influence of coastal upwelling-downwelling variability on tropical euphausiid abundance and community structure in the inshore Mexican central Pacific. Mar. Ecol. Prog. Ser. 451: 119-136. http://dx.doi.org/10.3354/meps09607 distribuciones uniformes como consecuencia de la exclusión o competencia. Esto último ha sido observado por Landa-Jaime (2013) para C. purpurascens, especie piscívora que presenta una distribución espacial uniforme. En el caso de C. prínceps, se observaron los 3 tipos de distribución espacial, pero la distribución de tipo agregada fue la más frecuente. Esta distribución podría explicarse por su preferencia alimenticia ya que, aunque no se le ha podido observar alimentando en laboratorio o campo, la forma de su rádula sugiere que se alimenta de poliquetos (Ortíz-Arellano 2014). Por lo tanto, los caracoles podrían estar agregados en torno a microambientes que presentan condiciones óptimas para el desarrollo de sus presas. Dado que los muestreos se realizaron a lo largo de un periodo de tiempo amplio, los cambios observados en el patrón de distribución entre los sitios de muestreo pudieran ser explicados a partir de variaciones conductuales de los caracoles.

Con respecto a la afinidad con el sustrato, se observó que C. princeps muestra una mayor predilección por habitar en sustratos de roca con algas (68\%). Ríos-Jara et al. (2006) mencionaron que esta especie se encuentra asociada a sustratos rocosos y roca con alga. Landa-Jaime (2013) resaltó 3 sustratos preferidos por C. princeps, de los cuales el sustrato coralino fue donde registró la mayor presencia (57\%); no obstante, su trabajo fue realizado precisamente en el arrecife coralino de Tenacatita. Habría que considerar que el sustrato preferido podría estar determinado por la disponibilidad de presas, por la facilidad de pasar desapercibidos durante la caza, o bien, por la facilidad de evitar ser depredados. Para evitar un sesgo en la selección del sitio de muestreo, se decidió realizar el estudio considerando varios sitios a lo largo de un gradiente latitudinal y con diferentes características; por tanto, se considera que los resultados obtenidos son representativos de la población en la costa de Jalisco.

La información generada con este trabajo podrá apoyar al conocimiento existente sobre los caracoles C. princeps. No obstante, es necesario continuar con estudios sobre la biología de esta especie, ya que aspectos como su época reproductiva, crecimiento, reclutamiento, o precisa forma de alimentación, entre otros, son factores relevantes que han sido poco estudiados y que permitirán, junto con los estudios específicos sobre toxicología, profundizar un poco más en el conocimiento de este recurso natural.

Un punto que resalta es que después de realizar actividades de campo en 13 sitios a lo largo de la costa sur de Jalisco y considerando 3 ambientes y un rango batimétrico entre los 0 y $20 \mathrm{~m}$ de profundidad, solo se lograron recolectar 180 especímenes vivos de C. princeps, lo que indica que se trata de una especie escasa o difícil de localizar. Esto llevaría a reflexionar sobre la pertinencia de establecer medidas de conservación de largo plazo, tales como restricciones de recolecta de la especie o creación de zonas de reserva o parques nacionales. En el caso de los caracoles del género Conus, se ha avanzado en el conocimiento de las propiedades de sus toxinas y sus aplicaciones biomédicas; sin embargo, también se deben estudiar aspectos básicos 
Arriaga-Cabrera L, Vázquez-Domínguez E, González-Cano J, Jiménez-Rosenberg R, Muñoz-López E, Aguilar-Sierra V (coordinators). 1998. Regiones marinas prioritarias de México. Mexico: Comisión Nacional para el Conocimiento y Uso de la Biodiversidad; [accessed $2018 \mathrm{Jul}$ 14]. http://www. conabio.gob. $\mathrm{mx} /$ conocimiento/regionalizacion/doctos/marinas.html.

Bandyopadhyay PK, Stevenson BJ, Ownby JP, Cady MT, Watkins M, Olivera BM. 2008. The mitochondrial genome of Conus textile, coxI-coxII intergenic sequences and Conoidean evolution. Mol. Phylogenet. Evol. 46(1): 215-223. https://doi.org/10.1016/j.ympev.2007.08.002

Bastida-Izaguirre D. 2014. Inventario, diagnóstico ambiental y ordenamiento espacial marino del santuario islas e islotes de Bahía de Chamela, Jalisco, México [dissertation]. [Zapopan (Jalisco, Mexico)]: Universidad de Guadalajara; 188 pp.

Bernáldez J, Jiménez S, González LJ, Noda-Ferro J, Soto E, Salceda E, Chávez D, Aguilar MB, Licea-Navarro A. 2016. A new member of gamma-conotoxin family isolated from Conus princeps displays a novel molecular target. Toxins 8(2): 1-18. http://dx.doi.org/10.3390/toxins8020039

Cervantes-Henández P, Michel-Morfín JE, Gallardo-Berumen MI. 2016. Reproductive and recruitment seasons of the purple snail Plicopurpura pansa (Gould, 1853) in Oaxaca, Mexico. J. Shellfish Res. 35(4): 993-1005. https://doi.org/10.2983/035.035.0422

Cortez-Orozco DM. 2017. Distribución, abundancia y preferencia de hábitat de los caracoles marinos del género Conus del submareal de la costa de Jalisco [BSc thesis]. [Autlán de Navarro (Jalisco, Mexico)]: Universidad de Guadalajara; 63 pp.

Dance SP, von Cosel R. 1977. Das grosse Buch der Meeresmuscheln: Schenecken und Muscheln der Weltmeere. Stuttgart (Germany): Ulmer Eugen Verlag; 304 pp.

Díaz-M JM, Gracia-C AM, Cantera-K JR. 2005. Checklist of the cone shells (Mollusca: Gastropoda: Neogastropoda: Conidae) of Colombia. Biota Colombiana 6(1): 73-86.

Duda TF Jr, Kohn AJ. 2005. Species-level phylogeography and evolutionary history of the hyperdiverse marine gastropod genus Conus. Mol. Phylogenet. Evol. 34(2): 257-272. https://doi.org/10.1016/j.ympev.2004.09.012

[EMA] European Medicines Agency. 2014. Prialt ziconotide. Epar summary for the public.London (UK): EMA; [2018 Jul 16] http://www.ema.europa.eu/docs/en_GB/document_library/ EPAR_Summary_for_the_public/human/000551/

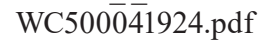

ESRI. 2011. ArcGIS Desktop: Release 10. Redlands (CA): Enviromental Systems Research Institute.

Flores-Garza R, García-Moctezuma YM, Flores-Rodríguez P, Michel-Morfín JE, Torreblanca-Ramírez C. 2014. The Conidae family (snail producers of poisons) associated with the rocky intertidal zone of Acapulco, Mexico. Nat. Res. 5(8): 343-350. http://dx.doi.org/10.4236/nr.2014.58032

García-Hernández VC. 2014. Análisis de la biodiversidad en arrecifes rocosos en la zona de transición Tropical-Subtropical del Pacífico mexicano [dissertation]. [La Paz (Baja California Sur, Mexico)]: Centro de Investigaciones Biológicas del Noroeste; $173 \mathrm{pp}$.

Gorrostieta-Hurtado E, Aguilar-Ramírez MB, Falcón-Alcántara A, Michel-Morfín E, Landa-Jaime V, Heimer de la Cotera EP. 2012. Perfil cromatográfico y efectos del extracto del conducto venenoso de Ductoconus princeps en Planorbella tenue. Bol. Soc. Int. Malacol. Med. Aplic. 18: 1-4.

Keen MA. 1971. Sea shells of tropical west America. Marine mollusks from Baja California to Peru. Second edition. Stanford (CA): Stanford University Press; 1069 pp. como el número real de especies en aguas mexicanas, su distribución, su abundancia y la biología básica de sus poblaciones.

\section{Agradecimientos}

Este estudio contó con financiamiento del Proyecto de Fondos Mixtos CONACYT-gobierno del estado de Jalisco (no. 2009-05-124426). GM recibió una beca tesis de Licenciatura del mismo fondo. Se agradece el apoyo en las actividades de campo de Israel Muñiz, Daniel Kosonoy y Álvaro Reyes Espinoza. Se agradecen las valiosas observaciones de los revisores anónimos.

Kohler KE, Gill SM. 2006. Coral Point Count with Excel extensions (CPCe): A Visual Basic program for the determination of coral and substrate coverage using random point count methodology. Computers \& Geosciences 32(9): 1259-1269. https://dx.doi.org/10.1016/j.cageo.2005.11.009

Kohn AJ. 1956. Piscivorous gastropods of the genus Conus. Proc. Natl. Acad. Sci. USA 42(3): 168-171. https://doi.org/10.1073/pnas.42.3.168

Kohn AJ. 1990. Tempo and mode of evolution in Conidae. Malacologia 32: 55-67.

Kohn AJ, Anderson T. 2010. The Conus Biodiversity website. Seattle (WA, USA): Burke Museum of Natural history and Culture [2014 Jun 12] http://biology.burke.washington.edu/conus/

Landa-Jaime V. 2013. Bases ecológicas de los caracoles marinos del género Conus asociados al arrecife coralino de Tenacatita, Jalisco, México [dissertation]. [Xalisco (Nayarit, Mexico)]: Universidad Autónoma de Nayarit; 217 pp.

Landa-Jaime V, Michel-Morfín E, Arciniega-Flores J, CastilloVargasmachuca S, Saucedo-Lozano M. 2013. Moluscos asociados al arrecife coralino de Tenacatita, Jalisco, en el Pacífico central mexicano. Rev. Mex. Biod. 84(4): 1121-1136. htpp://dx.doi.org/10.7550/rmb.32994

López J. 2001. Conotoxinas. Spira. 1: 7-11.

Marshall J, Kelley WP, Rubakhin SS, Bingham JP, Sweedler JV, Gilly WF. 2002. Anatomical correlates of venom production in Conus californicus. Biol. Bull. 203(1): 27-41. https://doi.org/10.2307/1543455

Medina-Elizalde J. 2010. Descripción morfológica de los órganos de mayor importancia de Conus (Conus) princeps var. Lineolatus (Linneo 1758 y Conus (Conus) brunneus (Wood 1828) [Bsc thesis]. [Puerto Ángel (Oaxaca, Mexico)]: Universidad del Mar; $72 \mathrm{pp}$.

Medina-Elizalde J. 2012. Efecto del veneno de Conus princeps y Anthopleura sp. sobre el canal TRPV1 [MSc thesis]. [Ensenada (Baja California, Mexico)]: Centro de Investigaciones Científicas y de Educación Superior de Ensenada; 87 pp.

Michel-Morfín E, Landa-Jaime V, Arciniega-Flores J. 2014. Moluscos bentónicos de fondos blandos de la plataforma continental del sur de Jalisco y Colima. In: Godínez-Domínguez E, Franco-Gordo C (eds.), Inventario de biodiversidad de la costa sur de Jalisco y Colima, vol 2. Autlán de Navarro (Jalisco, Mexico): Universidad de Guadalajara; pp. 14-32.

Michel-Morfín JE, Chavez EA, Landa-Jaime V. 2000. Population parameters and dye yield of the purple snail Plicopurpura pansa (Gould 1853) of west central Mexico. J. Shellfish Res. 19(2): 919-925. 
Morales-González D, Flores-Martínez E, Zamora-Bustillos R, Rivera-Reyes R, Michel-Morfín JE, Landa-Jaime V, Falcón A, Aguilar MB. 2015. Diversity of A-conotoxins of three wormhunting cone snails (Conus brunneus, Conus nux, and Conus princeps) from the Mexican Pacific coast. Peptides 68: 25-32. https://dx.doi.org/10.1016/j.peptides.2015.02.002

Muñiz-Castillo AI. 2014. Aspectos ecológicos de caracoles marinos toxoglosos (superfamilia Conoidea Fleming, 1822) de Carrizales, Colima [BSc thesis]. [Autlán de Navarro (Jalisco, Mexico)]: Universidad de Guadalajara; 75 pp.

Nybakken J. 1970. Radular anatomy and systematics of the West American Conidae (Mollusca, Gastropoda). Am. Museum Novitates 2414: 1-29, figs. 1-45.

Ortíz-Arellano MA. 2014. Diversidad, ecología y anatomía de los gasterópodos marinos de interés neurotóxico de la costa de Sinaloa, México [dissertation]. [Xalisco (Nayarit, Mexico)]: Universidad Autónoma de Nayarit; 249 pp.

Ponder WE. 1973. The origin and evolution of the Neogastropoda. Malacologia 12: 295-338.

Ríos-Jara E, Pérez-Peña M, López-Uriarte E, Enciso-Padilla I, Juárez Carrillo E. 2006. Biodiversidad de moluscos marinos de la costa de Jalisco y Colima, con anotaciones sobre su aprovechamiento en la región. In: Jiménez-Quiroz MC, EspinoBarr E (eds.), Los recursos Pesqueros y Acuícolas de Jalisco, Colima y Michoacán. Mexico: Instituto Nacional de la Pesca; p. $103-120$.
Silva-Segundo CA, Funes-Rodríguez R, Hernández-Rivas ME, Ríos-Jara E, Robles-Jarero EG, Hinojosa-Medina A. 2008. Asociaciones de larvas de peces en relación a cambios ambientales en las Bahías de Chamela, Jalisco y Santiago, Manzanillo, Colima (2001-2002). Hidrobiológica. 18 (Sup.1): 89-103.

Wilkinson T, Wiken E, Bezaury Creel J, Hourigan T, Agardy T, Herrmann H, Janishevski L, Madden C, Morgan L, Padilla M. 2009. Ecorregiones Marinas de América del Norte. Montreal: Comisión para la Cooperación Ambiental; 200 p.

Wyrtki K. 1965. Surface currents of the Eastern Tropical Pacific ocean. Inter-Amer. Trop. Tuna Comm. Bull. 9: 271-304.

WoRMS. 2018. World Register of Marine Species. Ostende (Belgium): Flanders Marine Institute [accessed 2018 May 27]. http://www.marinespecies.org.

Zamora-Bustillos R, Rivera-Reyes R, Aguilar MB, Michel-Morfín E, Landa-Jaime V, Falcón A, Heimer EP. 2014. Identification, by RT-PCR, of eight novel I2-conotoxins from the wormhunting cone snails Conus brunneus, C. nux and C. princeps from the eastern Pacific (México). Peptides 53: 22-29. https://dx.doi.org/10.1016/j.peptides.2014.01.018

Zar JH. 2010. Biostatistical Analysis. 5th Ed. New Jersey: Prentice Hall; 944 pp.

Received October 2018, accepted June 2019. 